\title{
Fourier Transform Rheology: A New Tool to Characterize Material Properties
}

\author{
Massimiliano Grosso ${ }^{1}$ and Pier Luca Maffettone ${ }^{2}$ \\ ${ }^{1}$ Dipartimento di Ingegneria Chimica e Materiali, Università degli Studi di Cagliari \\ Piazza D'Armi, I-09123, Cagliari, \\ 2Dipartimento di Ingegneria Chimica, Università degli Studi di Napoli "Federico II" \\ Piazzale Tecchio, I-80125, Napoli \\ Italy
}

\section{Introduction}

Liquid multiphase systems such as polymer blends or emulsions are ubiquitous in many applications, including plastic production, food processing, pharmaceutical and cosmetic production. When the constituents of the multiphase system are incompatible the phases are immiscible, and, depending on their relative amount, the microstructure can consist of droplets in a matrix, elongated fibrils or a co-continuous structure (Utracki, 2003) as schematically shown in Figure 1. The morphology of the liquid multiphase system is important in the applications as it strongly affects processing properties, and the properties of the final products. With the term "morphology" we here indicate not only the overall form or shape of the physical structure of the system, but also the distribution and orientation of the phases, the interfacial area, and the volume of the interphase.

Hence, a profound knowledge of the relation between processing parameters, material properties and morphology is essential to optimize the performances of the liquid multiphase systems.
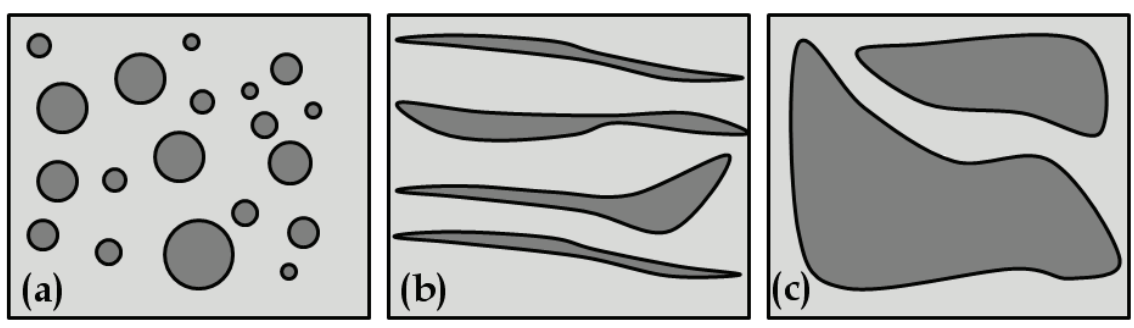

Fig. 1. Different morphologies of immiscible polymer blends (a) dilute droplet blends; (b) elongated fibrils; (c) co-continuous structure

Substantial efforts were done in the last decades to set up experimental protocols aimed at evaluating the morphological properties of polymer blends and emulsions via rheological measurements. So far, the most reliable strategy for morphological characterization through rheological measurements is based on the dynamic small amplitude oscillatory shear 
(SAOS) experiment: the samples are subjected to small amplitude shearing oscillations, and the measured shear stress response is used to gain information on the blend properties (e.g. Palierne, 1990).

Here, we present an alternative technique we have recently proposed to characterize the liquid two-phase system morphology. This methodology is based on Large Amplitude Oscillatory Shear (LAOS) flows. This kind of analysis is often referred in the literature as Fourier Transform Rheology (FTR) (Wilhelm et al., 1998), since the stress response is usually analyzed in the Fourier domain.

It will be shown that Fourier Transform Rheology possesses a high sensitivity in the characterization of the morphology, thus allowing evaluation of properties that might otherwise be hardly appreciated with traditional linear methodologies.

\section{Rheological oscillatory experiences}

\subsection{Small Amplitude Oscillatory Shear}

A typical tool used for the characterization of complex liquids is based on oscillatory rheometry (Macosko, 1994). The basic working principle of an oscillatory rheological test is to impose a sinusoidal shear deformation, and measuring the resultant shear stress response. In a typical experiment, the sample is placed between two plates (or a cone and plate geometry) (Figure 2): while the bottom plate remains stationary, a rotation is imposed on the top plate, thereby allowing a time-dependent strain deformation on the sample:

$$
\gamma(t)=\gamma_{0} \sin \omega t
$$

where $\omega$ is the oscillation frequency and $\gamma_{0}$ is the strain amplitude. The oscillation period is thus $\mathrm{T}=2 \pi / \omega$. The resulting time-dependent shear stress, $\sigma(\mathrm{t})$, is quantified by measuring the torque on the top plate. At low strain amplitudes, the stress response can be assumed to depend linearly on the strain deformation:

$$
\sigma(t)=A \sin \omega t+B \cos \omega t=\sigma_{0} \sin (\omega t+\delta)
$$

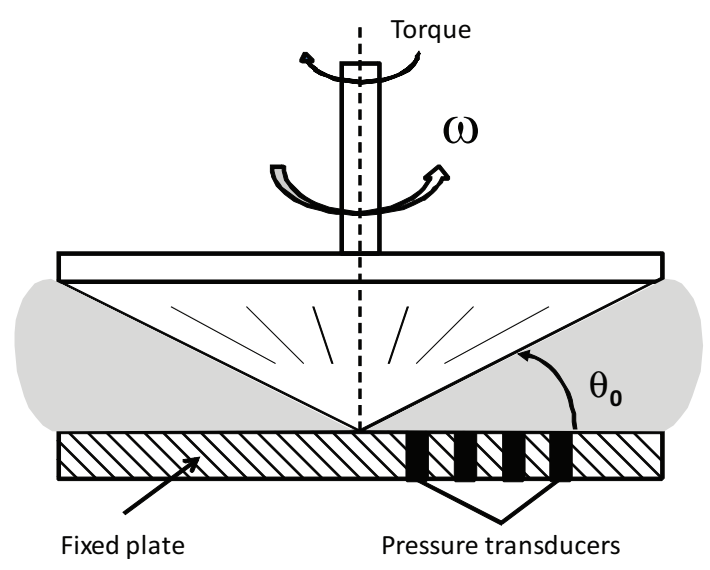

Fig. 2. Cone and plate geometry 
For this reason Small Amplitude Oscillatory Shear (SAOS) tests are usually referred as linear rheological measurements. If the material behaves as an ideal elastic solid, then the stress is in phase with the imposed deformation wave (i.e. proportional to $\sin (\omega t))$, and the proportionality constant is the shear modulus of the material. On the other hand, if the material is a purely viscous fluid, the stress is proportional to the rate of the strain deformation (i.e. proportional to $\cos (\omega t))$, and the proportionality constant is the viscosity of the fluid. The applied strain and the measured stress are in this case out of phase with phase angle $\delta=\pi / 2$.

Complex materials usually show a response that contains both in-phase and out-of-phase contributions. As a consequence, the total stress response at a given $\omega$ is characterized by both the sine and cosine components:

$$
\sigma(t)=G^{\prime}(\omega) \gamma_{0} \sin \omega t+G^{\prime \prime}(\omega) \gamma_{0} \cos \omega t
$$

In equation $3, G^{\prime}(\omega)$ is the storage modulus which characterizes the solid-like behavior, whereas $G^{\prime \prime}(\omega)$ is the loss modulus that takes into account the fluid-like contributions. The complex modulus to the frequency can be thus defined:

$$
G^{*}(\omega)=G^{\prime}(\omega)+i G^{\prime \prime}(\omega)
$$

where $i$ is the imaginary unit. Using the relation between the complex modulus $G^{*}(\omega)$ and the complex viscosity $\eta^{*}(\omega)$,

$$
G^{*}(\omega)=\omega \eta^{*}
$$

and

$$
\eta^{*}(\omega)=\eta^{\prime}+i \eta^{\prime \prime}
$$

The absolute value of the complex viscosity is of course given by:

$$
\left|\eta^{*}(\omega)\right|=\frac{\left|G^{*}\right|}{\omega}=\frac{\sqrt{G^{\prime 2}+G^{\prime \prime 2}}}{\omega}
$$

The frequency dependence of $G^{\prime}$ and $G^{\prime \prime}$ provides some important information about the microstructure of a material. For example, gels exhibit $G^{\prime}$ that is larger than $G^{\prime \prime}$ with both moduli independent of frequency. Polymer melts show $G^{\prime}$ and $G^{\prime \prime}$ at low frequencies that are dependent on $\omega^{2}$ and $\omega$, respectively. For viscoelastic materials, the overlap frequency (the frequency at which $G^{\prime}$ and $G^{\prime}$ curves intersect) gives information about the relaxation time of the system. The plateau modulus, i.e. the value of $G^{\prime}$ at high frequency, gives information about the strength of the structures formed in the material.

For the case of dilute blends with Newtonian constituents, the dependence of $G^{\prime}$ and $G^{\prime \prime}$ can be described in terms of the Palierne model which may quantitatively associate the linear viscoelastic properties of polymer blends to its chemical-physical properties e.g. to the interfacial tension (Palierne, 1990: Graebling et al., 1993a; Graebling et al., 1993b; Lacroix et al., 1996). 


$$
G^{*}(\omega)=G_{m}^{*}(\omega) \frac{1+3 \varphi H\left(\bar{R}_{V}, \omega\right)}{1-2 \varphi H\left(\bar{R}_{V}, \omega\right)}
$$

where

$$
\begin{gathered}
4\left(\frac{\alpha}{\bar{R}_{V}}\left(2 G_{m}^{*}(\omega)+5 G_{i}^{*}(\omega)\right)+\right. \\
H\left(\bar{R}_{V}, \omega\right)=\frac{\left(G_{i}^{*}(\omega)-G_{m}^{*}(\omega)\right)\left(16 G_{m}^{*}(\omega)+19 G_{i}^{*}(\omega)\right)}{40 \frac{\alpha}{\bar{R}_{V}}\left(G_{m}^{*}(\omega)+G_{i}^{*}(\omega)\right)+} \\
\left(2 G_{i}^{*}(\omega)+3 G_{m}^{*}(\omega)\right)\left(16 G_{m}^{*}(\omega)+19 G_{i}^{*}(\omega)\right)
\end{gathered}
$$

and $\mathrm{G}^{*}(\omega), \mathrm{G}^{*} \mathrm{~m}(\omega)$ and $\mathrm{G}^{*}(\omega)$ are, respectively, the complex moduli of the dispersed phase, matrix and blend at frequency $\omega, \alpha$ is the interfacial tension, $\phi$ is the volume fraction of the dispersed phase and $\bar{R}_{V}$ is the volume average drop radius of the included phase:

$$
\bar{R}_{V}=\frac{\int_{0}^{\infty} R^{4} \psi(R) d r}{\int_{0}^{\infty} R^{3} \psi(R) d r}
$$

where $\psi(R)$ represents the drop size distribution.

An example is reported in figure 3, where the elastic modulus for a blend composed by Poly-DiMethylSiloxane (PDMS) in Poly-IsoButylene (PIB) is reported with respect to the oscillation frequency, together with the elastic modulus of the neat constituents.

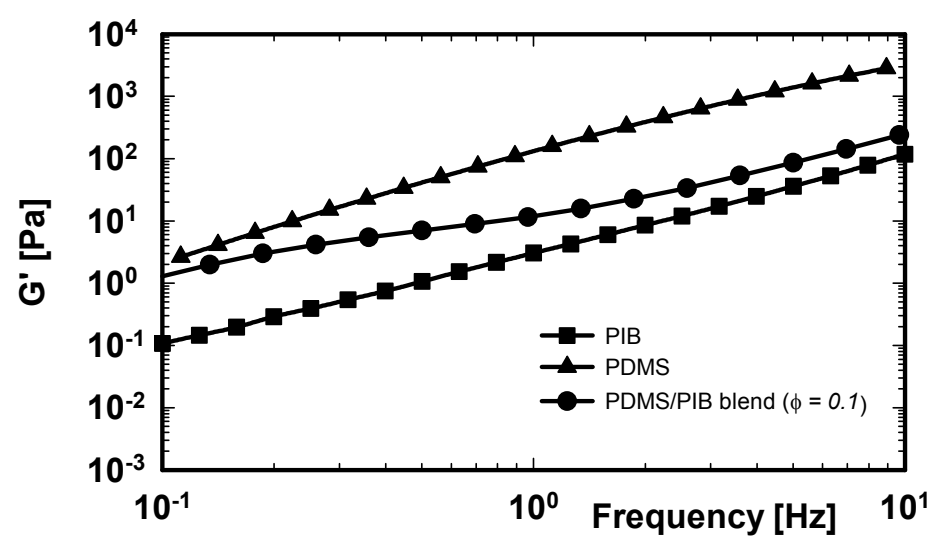

Fig. 3. Elastic modulus as a function of frequency for the pure components (PIB: square points; PDMS: triangles) and the polymer blend (full circles). The temperature is $30^{\circ} \mathrm{C}$. The strain amplitude is $50 \%$ 
The Palierne model is widely used to extract morphological or dynamical properties from oscillatory data, for example the average drop radius can be estimated (e.g. Das et al., 2005) or alternatively the surface tension (e.g. Huitric et al., 1998; Vincze-Minya and Schausberger, 2007). As already remarked in the literature (Graebling et al. 1993a), however, it is difficult to achieve a more detailed description of the morphology of the included phase. Indeed, a complete description of the drop size distribution cannot be reliably obtained with this technique. To our knowledge, the size distribution inference for a polymer blend based on the Palierne method has been carried out only by Friedrich et al. (1995). The methodology there proposed is based on a Tikhonv regularization, and gave satisfactory results only for very dilute blends with unimodal drop radius distributions.

\subsection{Large Amplitude Oscillations}

The above mentioned limits of the traditional linear oscillatory experiences motivated the study of alternative experimental techniques that might be more sensitive to the material morphology. In this regard, Large Amplitude Oscillatory Shear flows (LAOS) proved to be a possible candidate for the morphological characterization. The capabilities of this technique to pinpoint nonlinear material characteristics have been already analyzed in other contexts (Neidhofer et al., 2004; Schlatter et al., 2005), proving to be quite effective.

In the following we will briefly review some basic issues on LAOS (see Wilhelm et al. 1999 for details). When dealing with LAOS flows, the nonlinear dependences of the viscosity on the applied shear rate $\eta(\dot{\gamma})$ can be expected to be important, and equation 3 is no longer valid.

Due to the symmetry properties, the viscosity is independent of the shear direction and therefore it can only depend on the absolute shear rate (Wilhelm et al., 1999):

$$
\eta(\dot{\gamma})=\eta(-\dot{\gamma})=\eta(|\dot{\gamma}|)
$$

The Taylor expansion for the viscosity at small shear rates is given in equation (12):

$$
\eta(\dot{\gamma})=\eta_{0}+\eta_{1}|\dot{\gamma}|+\eta_{2}|\dot{\gamma}|^{2}+\ldots
$$

If the applied shear deformation is a harmonic oscillation with a given frequency $\omega_{1}$, strain and strain rate are:

$$
\gamma=\gamma_{0} \sin \omega_{1} t \quad \Rightarrow \quad|\dot{\gamma}|=\omega_{1} \gamma_{0}\left|\cos \omega_{1} t\right|
$$

Therefore, the absolute value of the shear rate signal $|\dot{\gamma}|$ can be represented in terms of a proper Fourier series (Ramirez, 1985):

$$
\begin{gathered}
|\dot{\gamma}|=\omega_{1} \gamma_{0}\left(\frac{2}{\pi}+\frac{4}{\pi}\left(\frac{\cos 2 \omega_{1} t}{1 \cdot 3}-\frac{\cos 4 \omega_{1} t}{3 \cdot 5}+\ldots\right)\right) \\
|\dot{\gamma}|=a^{\prime}+b^{\prime} \cos 2 \omega_{1} t+c^{\prime} \cos 4 \omega_{1} t+\ldots
\end{gathered}
$$


By substituting Equation 15 into Newton's equation for the viscosity leads to equation 16 (Wilhelm et al., 1999)

$$
\begin{gathered}
\sigma=\eta(\dot{\gamma}) \dot{\gamma}=\left(\eta_{0}+\eta_{1}|\dot{\gamma}|+\eta_{2}|\dot{\gamma}|^{2}+\ldots\right) \cos \omega_{1} t \\
\sigma=\eta(\dot{\gamma}) \dot{\gamma}=\left(\begin{array}{l}
\eta_{0}+\eta_{1}\left(a^{\prime}+b^{\prime} \cos 2 \omega_{1} t+\ldots\right)+ \\
\eta_{2}\left(a^{\prime}+b^{\prime} \cos 2 \omega_{1} t+\ldots\right)^{2}+\ldots
\end{array}\right) \cos \omega_{1} t
\end{gathered}
$$

The terms in brackets in Equation 17 can be thus simplified and written as a sum of even harmonics:

$$
\sigma=\left(a^{\prime \prime}+b^{\prime \prime} \cos 2 \omega_{1} t+c^{\prime \prime} \cos 4 \omega_{1} t+\ldots\right) \cos \omega_{1} t
$$

By multiplying the terms in the brackets by $\cos \omega_{1}$ t one ends up with the shear stress expression depending only on odd harmonics:

$$
\sigma=A \cos \omega_{1} t+B \cos 3 \omega_{1} t+C \cos 5 \omega_{1} t+\ldots
$$

where A, B, C are complex numbers. In a last step the non-linear torque signal is analyzed towards frequency components by Fourier transformation. Eventually, the signal can be described in terms of an odd function of the sinusoidal deformation

$$
\begin{aligned}
& \sigma(t)=\sum_{\substack{k=1 \\
\text { oddk }}}^{\infty} I_{R k} \cos j \omega_{1} t+I_{I k} \sin j \omega_{1} t= \\
& \sum_{\substack{k=1 \\
\text { odd } k}}^{\infty} I_{A k} \cos \left(k \omega_{1} t+\phi_{k}\right)
\end{aligned}
$$

In equation (20) $I_{\mathrm{Rk}}, I_{\mathrm{Ik}}$ and $I_{\mathrm{Ak}}$ are real coefficients. Straightforwardly, one can easily express the measured shear stress signal in the Fourier domain as:

$$
\sigma(t) \stackrel{F T}{\Leftrightarrow} \tilde{\sigma}(\omega)=\int_{-\infty}^{+\infty} \sigma(t) e^{-i \omega t}=\sum_{\substack{k=-\infty \\ \text { oddk }}}^{k=\infty} I_{k} \delta\left(\omega-k \omega_{1}\right)
$$

In equation $21, \delta\left(\omega-\mathrm{k} \omega_{1}\right)$ is the Dirac delta located at $\omega=\mathrm{k} \omega_{1}(\mathrm{k} \in \mathrm{Z}), i$ is the imaginary unit, and $I_{\mathrm{k}}$ is the (complex) coefficient of the $k$-th harmonic. As the stress time series $\sigma(\mathrm{t})$ is real valued, the condition $I_{k}=I_{-k}^{*}$ (with * denoting the complex conjugate) holds. As a consequence of the assumption made in equation 19, only odd terms of the Fourier series could be in principle accounted for in equation 21 (Wilhelm et al., 1998). It is easy to show that the following relationship among the coefficients holds:

$$
I_{A j}=\left|I_{k}\right|=\sqrt{I_{R k}^{2}+I_{I k}^{2}}
$$

When dealing with SAOS flows only the first term of the summation in equation 21 is significant. Incidentally, one can notice that, as $\gamma_{0}$ tends to zero, the linear behaviour is recovered, thus $I_{\mathrm{I} 1}=\mathrm{G}^{\prime} \gamma_{0}$ and $I_{\mathrm{R} 1}=\mathrm{G}^{\prime \prime} \gamma_{0}$, and $I_{\mathrm{Rk}} \approx I_{\mathrm{Ik}} \approx 0$ for any $k>1$. The appearance of 
significant values for $I_{\mathrm{k}}(k>1)$ marks the onset of nonlinearities in the stress response. As a further remark, it has been shown that, at vanishing amplitudes the following scaling for intensity of nth harmonic with strain has been observed for the constitutive equations so far investigated (Nam et al. 2008; Yu et al. 2008):

$$
I_{A n} \propto \gamma_{0}^{n}
$$

This allows the definition of new scalars, based on the ratios of intensities of higher harmonics and first harmonic of the stress response. For example, coefficient $Q$ is defined as (Hyun \& Wilhelm, 2009)

$$
Q=\frac{I_{A 3}}{I_{A 1}} \frac{1}{\gamma_{0}^{2}}
$$

This coefficient has been claimed to be helpful in distinguishing molecular architecture of polymers based on LAOS (Hyun \& Wilhelm, 2009).

From a practical point of view, the measurement of the stress, through the torque sensor of the rheometer, is usually performed discretely at finite sample intervals $(\Delta t)$. Based on the sampling frequency, $(r=1 / \Delta t$, number of data points collected per second), we obtain a time series $\sigma(\mathrm{n})$ of discrete measurements collected at $N_{\mathrm{P}}$ instants.

Discrete Fourier transform of this time domain series will be a series of $N_{\text {ST }}$ complex numbers evaluated through well consolidated FT techniques (Bracewell, 1986):

$$
\Sigma(k)=\sum_{n=1}^{\infty} \sigma(n) e^{-\frac{i 2 \pi(k-1)(n-1)}{N}}, 1 \leq k \leq N
$$

The maximum frequency in the Fourier domain will correspond to the Nyquist frequency $=$ $2 \pi / \Delta \mathrm{t}$. With the property of the Fourier transform leading to meaningful $N / 2$ (symmetric) terms, the resolution in the frequency domain is $2 \pi / T$. Therefore, sampling interval determines maximum frequency to which information can be obtained, while the duration of measurements determines the resolution of frequency. Larger $\mathrm{T}$ values also lead to higher signal to noise ratio. It should be remarked that some techniques are introduced in the literature in order to improve the sensitivity to the signal of the measurement (Wilhelm et al., 1999).

\section{FTR on polymer blends}

\subsection{Theory}

In this section, we will focus on the theoretical aspects concerning the characterization through FTR of immiscible blends with low fraction of the dispersed phase. In this case the morphology of the included phase is globular: the basic element of such a dilute blend is thus a single drop dispersed in a matrix. Therefore, the study of single droplet behaviour is regarded as a reasonable starting point to model the complex behaviour of immiscible polymer blends. The overall rheological response, in fact, could be determined just on such a basis.

The dynamic behaviour of dilute polymer blends subjected to LAOS flows can be modeled as recently proposed in the literature by considering the single droplet dynamics together 
with a proper stress expression (Rallison, 1984; Stone, 1994; Almusallam et al., 2000; Yu et al., 2002; Jackson and Tucker, 2003; Yu and Bousmina, 2003). For what matters the dynamics of the drop, a handy though effective phenomenological model has been proposed by Maffettone and Minale (1998) and applies to generic flow fields. The model is formulated in terms of at most six first-order, ordinary, differential equations, and is capable of describing drop deformation up to the nonlinear range. This model is known to be quite accurate for small-to-medium droplet deformation, but loses some quantitative accuracy as droplet deformation becomes large. We use here this model for its simplicity, even though significant distortion of drop shape is expected under LAOS. Still, the Maffettone and Minale model provides a useful basis for analyzing and interpreting the experimental results also when significant strain deformations occur (Guido et al. 2004). The drop is described as an ellipsoid by a second rank symmetric, positive definite, and time dependent tensor. The shape dynamics can be thus described by the evolution of tensor $\mathbf{S}$ which follows the equation:

$$
\frac{d \mathbf{S}}{d t}-(\boldsymbol{\Omega} \cdot \mathbf{S}-\mathbf{S} \cdot \mathbf{\Omega})=-\frac{f_{1}}{\tau}\left(\mathbf{S}-\frac{3}{I_{2}} \mathbf{I}\right)+f_{2}(\mathbf{S} \cdot \mathbf{D}+\mathbf{D} \cdot \mathbf{S})
$$

In Equation 26, $\tau$ is the emulsion time $(\tau=\eta R / \Gamma)$ where $\eta$ is the matrix viscosity, $R$ the undistorted drop radius and $\Gamma$ is the interfacial tension; $I$ is the second rank unit tensor, $\mathbf{D}$ and $\Omega$ are the deformation rate and the vorticity tensors respectively, and $I_{2}$ is the second scalar invariant of tensor $\mathbf{S}$. The shear flows here considered give the following forms for the deformation and vorticity tensors:

$$
\mathbf{D}=\frac{1}{2} C a\left(\begin{array}{ccc}
0 & 1 & 0 \\
1 & 0 & 0 \\
0 & 0 & 0
\end{array}\right), \quad D=\frac{1}{2} C a\left(\begin{array}{ccc}
0 & 1 & 0 \\
-1 & 0 & 0 \\
0 & 0 & 0
\end{array}\right)
$$

In equation 27 the Capillary number is introduced:

$$
C a=\frac{\text { viscous stress }}{\text { interfacial stress }}=\frac{\eta \dot{\gamma}}{\Gamma / R}
$$

This gives the ratio between the two competing forces affecting the drop shape in shear flow experiences: the driving force of deformation (i.e. the shear stress), and the resistance force supporting the shape of the drop, that is the interfacial tension. The dependence of the capillary number on time is understood. The functions $f_{1}$ and $f_{2}$ appearing in Eq. 26 are given by (Maffettone \& Minale, 1998):

$$
\begin{aligned}
& f_{1}(\lambda)=\frac{40(\lambda+1)}{(2 \lambda+3)(19 \lambda+16)} \\
& f_{2}(\lambda)=\frac{5}{2 \lambda+3}+\frac{3 C a^{2}}{2+6 C a^{2}}
\end{aligned}
$$

In equation 29 the ratio $\lambda=\eta_{d} / \eta_{m}$ is defined, where $\eta_{d}$ is the viscosity of the dispersed phase and $\eta_{m}$ is the matrix viscosity. At rest the drop is spherical $(S=I)$. Notice finally that within this description drop break-up is absent under shear flow for $\lambda \geq 3$. 
Once the state of the drop deformation is known, one can calculate the stress $\boldsymbol{\sigma}$ of a dilute polymer blend according to Batchelor (1970):

$$
\begin{aligned}
& \boldsymbol{\sigma}=\underset{\substack{\text { isotropic } \\
\text { term }}}{-p \mathbf{I}}+\eta_{m}\left(\nabla \mathbf{v}+\nabla \mathbf{v}^{T}\right) \\
& -\frac{\eta}{V} \int_{S}(\mathbf{n u}+\mathbf{u n}) d A+\frac{\Gamma}{V} \int_{S}\left(\mathbf{n} \mathbf{n}-\frac{1}{3} \mathbf{I}\right) d A
\end{aligned}
$$

In Eq. 30, p is the pressure, $\nabla \mathbf{v}$ is the velocity gradient tensor and $\nabla \mathbf{v}^{\mathrm{T}}$ its transpose, $\eta$ is the viscosity of the continuous phase, $\mathrm{V}$ is the total volume of the system, $\mathbf{n}$ is the unit vector normal to the ellipsoid surface, representing the interface between the two phases, $\mathbf{u}$ is the velocity at the interface, $d A$ is the area of an interfacial element, and the integrals are calculated over the whole interface of the system, $S$. Equation 26 can be used to predict the stresses if $\mathbf{n}$ and $\mathbf{u}$ are known. Predictions are obtained by integrating equations (26) and (29) for the drop morphology. The elastic interfacial term in equation (30) is calculated as suggested by Almusallam et al. (2004). The viscous term in the interface stress is neglected.

Equation 30 is the sum of two conceptually different terms: the first one is due to the Newtonian matrix contribution and depends linearly on the velocity gradient, whereas the second term (the viscous and the elastic term) corresponds to the sum of interfacial contributions related to the entire drop population. The first part depends linearly on the applied shear rate, whereas the interfacial contribution is the only nonlinear term appearing in Equation 29. Under LAOS, the first term will not contribute to higher harmonics in the shear stress for its linear nature. Conversely, the interface contribution will give rise to higher harmonics in the power spectrum of $\sigma(t)$ (Grosso and Maffettone, 2007). Consequently, the contribution to the higher harmonics in the Fourier spectrum of each drop with radius $R$ is directly related to the interfacial contribution. In the frequency domain this can be written as:

$$
\begin{aligned}
& I_{k}(R)=\frac{2 \pi}{T} \int_{T} \sigma(t) e^{-i k \omega_{1} t} d t \\
& =-\frac{2 \pi}{T} \int_{T}\left(\frac{\eta}{V} \int_{A}(\mathbf{n u}+\mathbf{u n}) d A+\frac{\eta}{V} \int_{A}\left(\mathbf{n n}-\frac{1}{3} \mathbf{I}\right) d A\right) e^{-i k \omega_{1} t} d t
\end{aligned}
$$

with k odd and $>1$.

Simulations are performed by mimicking realistic conditions for PDMS in PIB samples with the relevant parameters reported in the Table 1 . These parameter values are consistent with experiments carried out at a temperature $\mathrm{T}=35^{\circ} \mathrm{C}$. The volume fraction is always assumed to be $\phi=0.1$.

Figure 4 shows the tangential stress $\sigma$ of two simulated polymer blends both in the time and in the Fourier domains. The imposed deformation amplitude and oscillation frequency are set respectively equal to $\gamma_{0}=800 \%$ and $\omega=0.1 \mathrm{~s}^{-1}$. The two blends differ for drop radius. Figure (4.a) and (4.b) show the time evolution and the Fourier transform (namely the absolute values of power spectrum rescaled with respect the fundamental harmonic, thus $I\left(\omega_{1}\right)=1$ ) of a polymer blend consisting of equal drops with radius $R_{1}=1 \mu \mathrm{m}$, respectively, 
whereas figure (4.c) and (4.d) refer to a blend with drop radius $R_{2}=5 \mu \mathrm{m}$. It is apparent that no significant difference can be appreciated in the time domain, the signals looking very close to a sinusoidal waveform in both cases. This result is not unexpected since, as already mentioned, the linear Newtonian matrix contribution dominates the response when observed in the time domain.

On the contrary, the nonlinear features appear more evidently in the Fourier domain. The principal harmonic (corresponding to the forcing frequency $\omega=0.1 \mathrm{~s}^{-1}$ ) is not reported entirely in order to magnify the harmonics appearing at higher frequencies. It is evident that Fourier analysis allows a clear detection of the nonlinearities that are otherwise not appreciable in the time domain. By comparing Fig. (4.b) with Fig. (4.d), it can also be noted the significant dependence of higher harmonics of the shear stress on drop size.

\begin{tabular}{|c|c|c|c|c|c|}
\hline Polymer & Formula & $\begin{array}{c}\text { Molecular } \\
\text { Weight } \\
{[\mathrm{Da}]}\end{array}$ & $\begin{array}{c}\text { Density } \\
{\left[\mathrm{Kg} / \mathrm{m}^{3}\right]}\end{array}$ & $\begin{array}{c}\text { Viscosity } \\
{[\mathrm{Pa} \cdot \mathrm{s}]}\end{array}$ & $\begin{array}{c}\text { Interfacial tension } \\
{[\mathrm{mN} / \mathrm{m}]}\end{array}$ \\
\hline PDMS & {$\left[-\mathrm{Si}\left(\mathrm{CH}_{3}\right)_{2} \mathrm{O}-\right]_{\mathrm{n}}$} & 200000 & 971 & 175 & 3 \\
\hline PIB & {$\left[-\mathrm{CH}_{2} \mathrm{C}\left(\mathrm{CH}_{3}\right)_{2}-\right]_{\mathrm{n}}$} & 1300 & 894 & 57 & \\
\hline
\end{tabular}

Table 1. Main physical properties of PDMS/PIB system
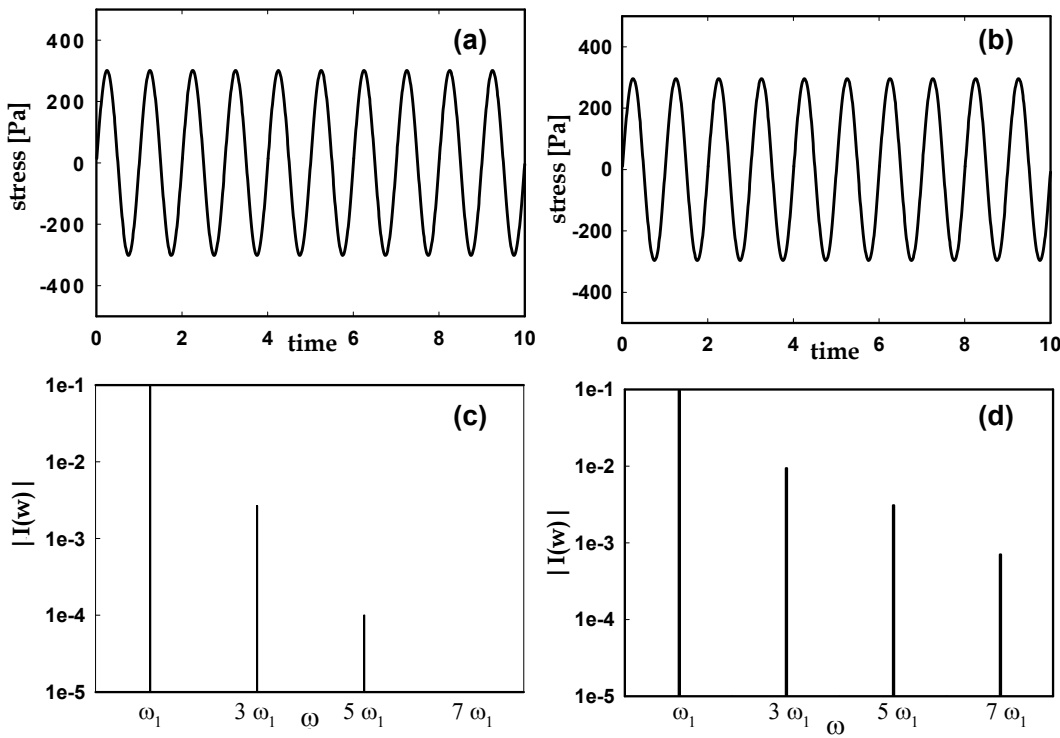

Fig. 4. Tangential stress of a simulated polymer blend in a LAOS experience in the time domain (Figures 4.a and 4.b) and in the Fourier domain (Figures 4.c and 4.d) for two different drop radii: $R_{1}=1 \mu \mathrm{m}$ (Figures 4.a and 4.c) and $R_{2}=5 \mu \mathrm{m}$ (Figures 4.b and 4.d). The deformation amplitude is $\gamma_{0}=800 \%$ and $\omega_{1}=0.1 \cdot 2 \pi \mathrm{s}^{-1}$. The physical parameters are in Tab. 1 
Figure 5 reports the absolute values $I_{3 \mathrm{~A}} / \gamma_{0}{ }^{3}$ and $I_{5 \mathrm{~A}} / \gamma_{0}{ }^{5}$ for a fixed value of the radius $R=10$ $\mu \mathrm{m}$ versus the strain amplitude $\gamma_{0}$. As the strain deformation tends to zero, both quantities approach a constant value thus confirming the asymptotic behaviour previously observed for other constitutive models (Nam et al. 2008; Ewoldt et al. 2008). It should be remarked that the limiting values depend on the blend properties (i.e. the phase viscosities, the surface tension and the drop radii).

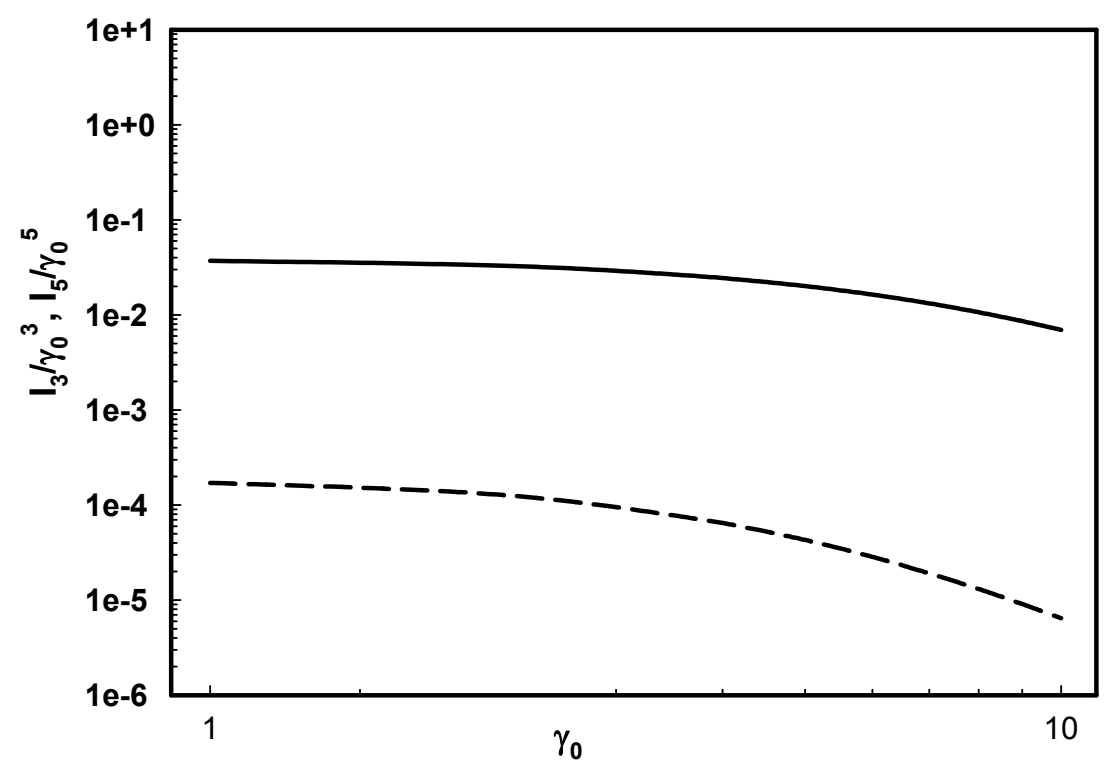

Fig. 5. Scalars $I_{3} / \gamma_{0}{ }^{3}$ and $I_{5} / \gamma_{0}{ }^{5}$ vs the strain deformation $\gamma_{0}$ for a simulated monodisperse polymer blend with radius $R=10 \mu \mathrm{m}$

\subsection{Experiments}

In this section we will show some experimental results that demonstrate the sensitivity of the FTR methodology when analyzing blend or emulsion morphology. The details of the experimental part can be found elsewhere (Carotenuto et al. 2008).

The polymer blend is prepared with PDMS and PIB that are immiscible at room temperature. PIB/PDMS emulsion is a widely used model system largely studied in the literature by means of both rheological and optical techniques (Jansseune et al., 2000; Guido et al. 2004; Wannaborworn et al. 2002). All the experiments were performed at constant temperature $\mathrm{T}=30^{\circ} \mathrm{C}$. The main physical properties of the polymers are reported in Table 1 . The value of the interfacial tension for the very same polymers is found in the literature (Sigillo et al., 1997). PIB is the continuous phase and PDMS is the dispersed phase. All the experiments were carried out with a volumetric fraction, $\phi$, of the dispersed phase fixed to 0.1 thus leading to a globular morphology. This value is small enough to consider coalescence negligible. The viscosity ratio is equal to 3 , and it is large enough to avoid 
significant break-up phenomena under pure shear flow. Thus, the blend can be assumed to be stable, and its microstructure should not significantly vary in time during the experiments (negligible breakup and negligible coalescence). The blend morphology, i. e., the drop size distribution $\psi(\mathrm{R})$, is then assumed to remain unchanged during LAOS experiments.

The experiments were conducted on three different blend samples, which hereafter will be indicated with a capital letter A, B and C. The morphologies of the samples are supposed to have a similar (but not equal) morphology since their preparation followed the same protocol.

Oscillatory shear measurements (both SAOS and LAOS) were performed in a conventional strain controlled rheometer (ARES, TA Instruments). Linear viscoelastic measurements were analyzed using the software provided by the rheometer manufacturer. LAOS experiments required a modification and improvement of the traditional rheometer data acquisition system. The raw data coming from both motor and transducer were acquired and digitized with a 16-bit analog-to-digital converter (National Instrument, PCI_6251). The motor signal was correlated to the imposed strain deformation, $\gamma$, while the transducer signal was associated with the measured torque. In order to maximize $\mathrm{S} / \mathrm{N}$, the rheometer was equipped with a very sensitive torque transducer (2KFRTN1) that could detect a torque ranging from 0.002 to $200 \mathrm{mN} \cdot \mathrm{m}$.

Before starting the acquisition, two main parameters were set: the scan rate, $\mathrm{r}[=] \mathrm{pts} / \mathrm{s}$, and the number of data points, $N_{\mathrm{p}}$. They were the same for both the channels (motor signal and transducer signal). The ratio between $N_{\mathrm{p}}$ and $r$ gives the time required for the entire acquisition, $t_{\mathrm{acq}}=N_{\mathrm{p}} / r$. The oscillation cycles collected during $t_{\mathrm{acq}}$ depend on the imposed deformation frequency $\left(\omega_{1}\right)$. Typical values of $r$ and $N_{\mathrm{p}}$ are $1000 \mathrm{pts} / \mathrm{s}$ and $80000 \mathrm{pts}$, respectively, thus $t_{\mathrm{acq}}=80 \mathrm{~s}$. Thus, for an imposed deformation frequency $\omega_{1}=0.1 \mathrm{~Hz}, 8$ complete cycles were acquired. It should be noted that the higher values of $r$ and $N_{\mathrm{p}}$, the higher the $\mathrm{S} / \mathrm{N}$ ratio (Wilhelm et al., 1999). It was however checked that acquisitions with larger amount of data $\left(r=5,000 \mathrm{pts} / \mathrm{s}\right.$ and $\left.N_{\mathrm{p}}=400,000 \mathrm{pts}\right)$ did not show any significant increase in the quality of our data.

Raw data coming from transducer were collected and subsequently transformed into the corresponding Fourier spectra. Odd multiples of the fundamental harmonic appear in the nonlinear regime (LAOS). For the polymer blend under investigation, the 3rd and the 5th overtones could be clearly detected in the shear stress Fourier spectrum for deformation amplitudes $\gamma 0>100 \%$. The electric signal measured by the torque transducer is supplied in terms of potential difference units.

LAOS data were analyzed according to the FTR protocols. The imposed sinusoidal deformation is $\gamma(t)=\gamma_{0} \sin \left(\omega_{1} t\right)$, where $\omega_{1}=2 \pi \Omega_{1}=2 \pi / T$ is the characteristic angular frequency with $T$ the oscillation period.

Linear viscoelastic measurements were carried out for a preliminary characterization of the microstructure of the samples. Oscillatory measurements were performed with frequency ranging from 0.1 to $10 \mathrm{~Hz}$. Strain amplitudes up to $50 \%$ gave shear stress responses well within the linear region. It was found that traditional SAOS measurements did not give a clear discrimination between different blends, and the $G^{\prime}$ curves for the three emulsions are almost overlapping, thus indicating that SAOS suggest that the three blends have similar morphologies. 


\begin{tabular}{|c|c|}
\hline Blend & $\bar{R}_{V}[\mu \mathrm{m}]$ \\
\hline $\mathrm{A}$ & 5.6 \\
\hline $\mathrm{B}$ & 6.7 \\
\hline $\mathrm{C}$ & 8.0 \\
\hline
\end{tabular}

Table 2. Average drop radii for the three blends estimated with the Palierne method

From SAOS measurements one can obtain an estimation of the average dimension of the dispersed phase, namely the volume-average drop radius, $\bar{R}_{V}$. According to Palierne (1990), one can estimate the volume average drop radius for the emulsions. Table 2 contains the values of the estimated average drop radii for the blends A, B, C. As expected, the volume averaged drop radius, $\bar{R}_{V}$, for the three samples is very similar. A more detailed description of blend morphology cannot be attained with the linear rheological measurements.

LAOS measurements were performed with $\gamma_{0} \geq 200 \%$, where nonlinearities in the response become clearly appreciable. A typical experimental result is shown in Fig. 6, where the tangential stress response is reported both in the time (Fig. 6.a) and in the frequency domain (Fig. 6.b) for $\gamma_{0}=800 \%$ and $\Omega_{1}=0.1 \mathrm{~Hz}$ (or, equivalently, $\omega_{1}=2 \pi 0.1 \mathrm{rad} / \mathrm{sec}$ ). Fourier spectra report the absolute value of the overtones, normalized with the first harmonic $\left(I_{\mathrm{k}} / I_{1}\right.$ or equivalently $\left.I_{\mathrm{k} 1}\right)$ as commonly done in the FTR literature (e.g. Wilhelm et al., 1998). The nonlinear shear stress response cannot be easily detected in the time domain, but the corresponding power spectrum clearly shows the occurrence of a third and a fifth peak.
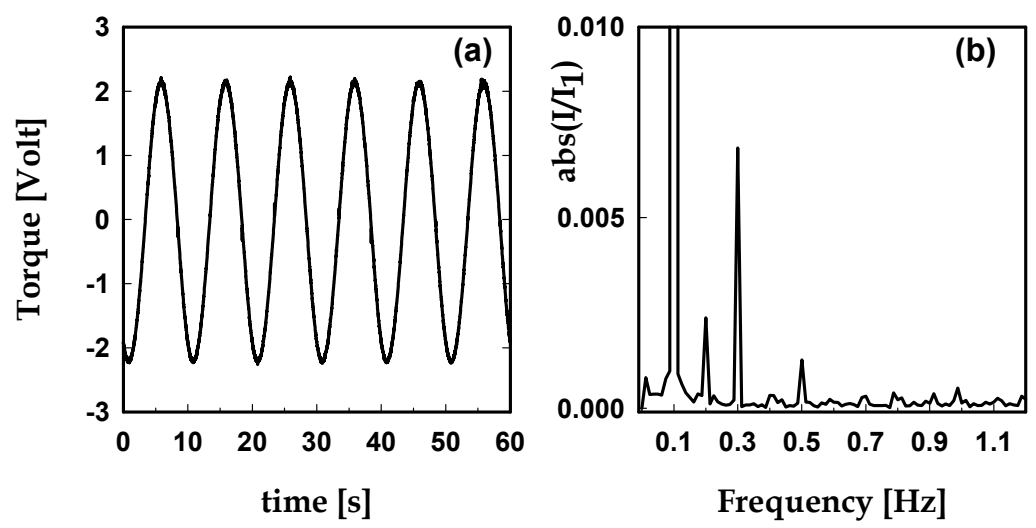

Fig. 6. Transducer signals in the time domain (a) and the corresponding Fourier spectra (b) for a polymer blend, at $30^{\circ} \mathrm{C}$

In figure $6 . \mathrm{b}$ a peak $I\left(2 \omega_{1}\right)$ at an even multiple of the fundamental harmonic is also observable. It should be reminded that this occurrence is unexpected since the stress signal is demonstrated to be an odd function of the time. Several explanations for the presence of even overtones in the spectra have been proposed in literature. Quite often, the occurrence 
of even harmonics is attributed to some artefact in the experiments as e.g. wall-slip phenomena (Hatzikiriakos \& Dealy, 1991). In the case here reported, the second harmonic seems to be material-independent, and it can be attributed to an imperfect alignment of the upper and lower plates of the rheometer (Carotenuto et al., 2008): it reasonably comes from the instrument itself and results unrelated to the measured sample, for this reason it is simply neglected.

Figure 7 shows the $Q$ coefficient defined in equation 24 as a function of the strain amplitude for the blend $\mathrm{C}$. The third harmonic is clearly detected for the polymer blend under investigation. The value of $I_{31}$ is small but reproducible with an experimental error lower than 3\%. For the sake of comparison, data of the neat PIB and PDMS are also reported in Fig. 7. The pure component $I_{31}$ is weighted by the corresponding amount in the blend (i.e., 0.1 for the PDMS and 0.9 for the PIB). It is apparent that the $I_{31}$ values of the pure components are extremely low, according to their quasi-Newtonian behavior, and negligible when compared with the $I_{31}$ values of the blend. This experimental evidence unequivocally suggests that the observed nonlinear response of the blend does not derive from simple superposition of the nonlinear contribution of the neat polymers, but it seems essentially due to the interface stress contribution. Such behaviour confirms the validity of the assumptions made in Equation 29.

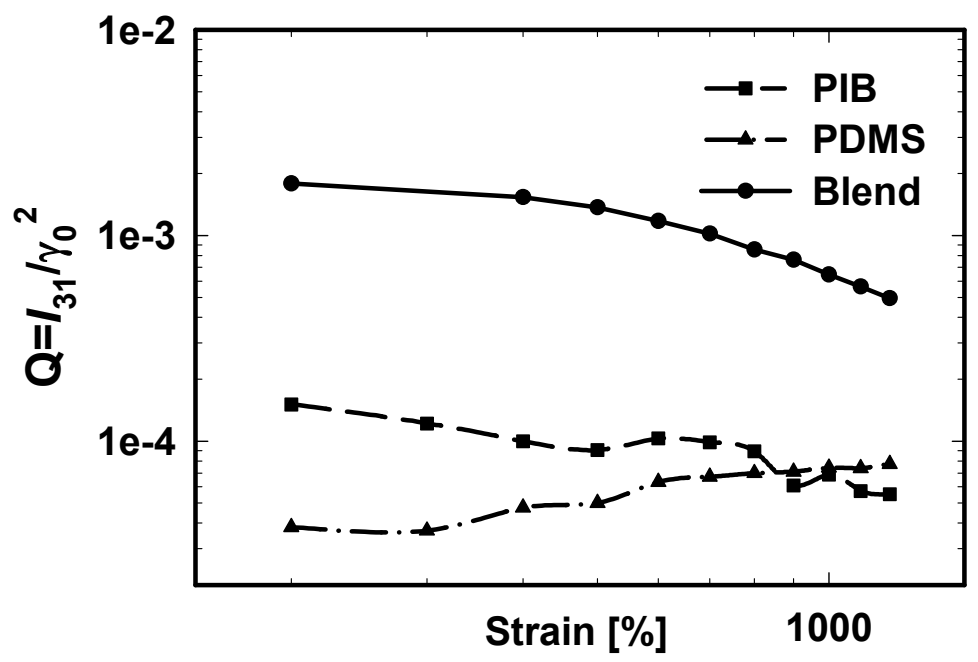

Fig. 7. The $Q$ coefficient as a function of the imposed strain deformation for the pure components (PIB: dashed line with square; PDMS: dashed-dotted line with triangles) and for the blend $\mathrm{C}$ (solid line with circles). The temperature is $30^{\circ} \mathrm{C}$. The oscillation frequency is $0.1 \mathrm{~Hz}$

Figure 8.a shows the coefficients $Q=I_{31} / \gamma_{0}{ }^{2}$ and $P=I_{51} / \gamma_{0} 4$ for the three blends $A, B$ and $C$ as a function of the strain amplitude. It is shown that, as the strain deformation decreases, the curves seem to tend to an asymptotic plateau value $Q_{0}$ and $P_{0}$, accordingly with the theoretical predictions. 
The curves do not superimpose, thus suggesting that LAOS experiences could discriminate between different morphologies. Indeed, as reported from Palierne results, the upper curve refers to the blend $\mathrm{A}\left(\bar{R}_{V}=5.6 \mu \mathrm{m}\right)$, the medium to the blend $\mathrm{B}\left(\bar{R}_{V}=6.7 \mu \mathrm{m}\right)$, and the lower to the blend $\mathrm{C}\left(\bar{R}_{V}=8 \mu \mathrm{m}\right)$. Hence, the $Q_{0}$ coefficient of the blend seems to decrease with the mean size of the inclusions. In Figure 8.b, the ratio $P=I_{51} / \gamma_{0} 4$ for the blends $A, B$ and $C$ are also reported. Since the fifth overtones are significantly smaller than the third ones, they are more affected by experimental noise. Analogously to $Q$ behaviour, the fifth peaks are larger for blend with smaller volume averaged drop radius.

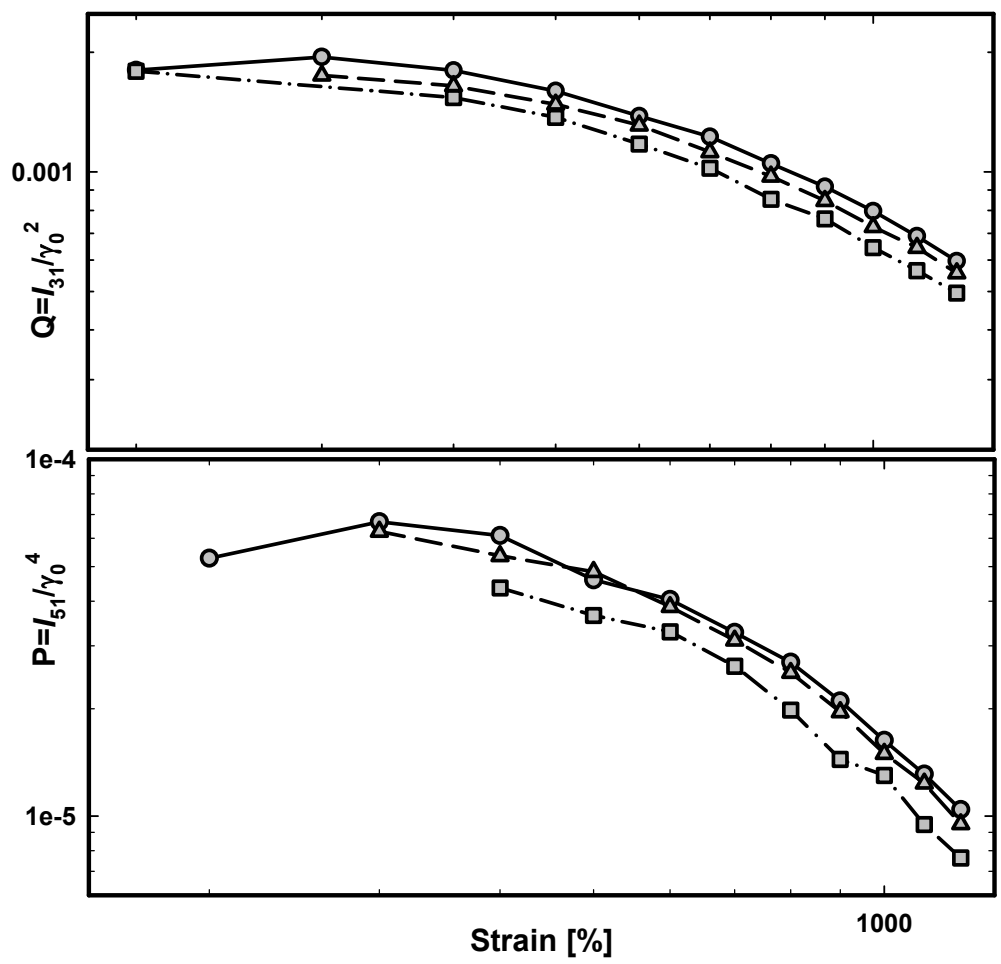

Fig. 8. The $Q$ and $P$ coefficients as a function of strain amplitude for the blend A (solid line with circles), $B$ (dashed line with triangles) and $C$ (dashed-dotted line with squares). The oscillation frequency is $0.1 \mathrm{~Hz}$

\section{Conclusion}

Fourier Transform Rheology is a valuable tool to characterize the microstructure of dilute immiscible polymer blend as it was shown both theoretically and experimentally. We analyze the case of a blend with Newtonian constituents, and in such a case the nonlinearity of the response under LAOS comes exclusively from the presence of a polymer-polymer 
interface. Indeed, distinct odd multiples of the fundamental harmonic are clearly evident in the power spectrum of the emulsion, while are only barely distinguishable in the spectra of the pure components (PIB and PDMS). FTR greatly enhances the sensitivity of the experiments to the blend morphology, thus allowing the evaluation of details that are otherwise difficult to be appreciated with time domain analysis.

\section{Acknowledgments}

Authors acknowledge Dr. Claudia Carotenuto for the helpful discussions on the experimental technique.

\section{References}

Almusallam A.S., Larson R. \& Solomon M.J. (2004). Comprehensive constitutive model for immiscible blends of Newtonian polymers, Journal of Rheology, 48(2), pp 319-348 ISSN: 0148-6055

Batchelor G.K. (1970) The stress system in a suspension of force-free particles, J. Fluid Mech. 41, pp 545-570 ISSN: 0022-1120

Bracewell R.N. (1986) The Fourier-transform and its application. McGraw-Hill, New York, ISBN: 978-0073039381

Carotenuto C., Grosso M., Maffettone P.L. (2008) Fourier Transform Rheology of Dilute Immiscible Polymer Blends: A Novel Procedure To Probe Blend Morphology, Macromolecules, 41, pp 4492-4500 ISSN: 0024-9297

Das N.C., Wang H, Mewis J. \& Moldenaers P. (2005), Rheology and Microstructures Formation of Immiscible Model Polymer Blends Under Steady State and Transient Flows, J. Pol. Sci.: Part B: Polymer Physics, 43, pp. 3519-3533 ISSN: 1099-0488

Ewoldt RH, Hosoi AE \& McKinley GH (2008) New measures for characterizing nonlinear viscoelasticity in large amplitude oscillatory shear. Journal of Rheology 52(6), pp. 1427-1458 ISSN: 0148-6055

Friedrich C., Gleinser W., Korat E. Maier D. \& Weese J. (1995). Comparison of sphere-size distributions obtained from rheology and transmission electron microscopy in PMMA/PS blend, Journal of Rheology, 39(6) pp. 1411-1425 ISSN: 0148-6055

Graebling D, Muller R. \& Palierne J.P. (1993). Linear viscoelastic behavior of some incompatible polymer blends in the melt - interpretation of data with a model of emulsion of viscoelastic liquids Macromolecules 26, pp. 320-329 ISSN: 0024-9297

Graebling D,, Muller R. \& Palierne J.P. (1993). Linear viscoelasticity of incompatible polymer blends in the melt in relation with interfacial properties, Journal de Physique $I V, 3$ pp. 1525-1534, ISSN: 1155-4339

Grosso M. \& Maffettone P.L. (2007) A new methodology for the estimation of drop size distributions of dilute polymer blends based on LAOS flows, J. Non Newt. Fluid Mech. 143, pp. 48-58 ISSN: 0377-0257

Guido S.; Grosso M.; Maffettone P.L. (2004). Newtonian drop in a Newtonian matrix subjected to large amplitude oscillatory shear flows, Rheologica Acta, 43, pp. 575-583 ISSN: 0035-4511

Hatzikiriakos, S. G., Dealy J. M. (1991) Wall slip of molten high density polyethylene. I. Sliding plate rheometer studies. Journal of Rheology 35(4), 497-523 ISSN: 0148-6055 
Huitric J., Mederic P., Moan M., \& Jarrin J. (1998) Infuence of Composition and morphology on rheological properties of polyethylene/polyamide blends, Polymer 39(20) pp. 4849-4856 ISSN: 0032-3861

Hyun K, Wilhelm M (2009) Establishing a New Mechanical Nonlinear Coefficient Q from FT-Rheology: First Investigation of Entangled Linear and Comb Polymer Model Systems. Macromolecules 42 pp. 411-422 ISSN: 0024-9297

Jansseune T.; Mewis J.; Moldenaers P.; Minale M. \& Maffettone P.L. (2000) Rheology and rheological morphology determination in immiscible two-phase polymer blends, $J$. Non-Newtonian Fluid Mech. 93, pp. 153-165 ISSN: 0377-0257

Lacroix C., Bousmina M., Carreau P.J., Favis B.D. \& Michel A.(1996) Properties of PETG/EVA blends .1. Viscoelastic, morphological and interfacial properties Polymer 37 (1996), pp. 2939 ISSN: 0032-3861

Macosko W. (1994) Rheology: Principles, Measurements and Applications, Wiley-VCH, New-York, ISBN: 978-0471185758

Maffettone P.L. and Minale M. (1998) Equation of change for ellipsoidal drops in viscous flows, J. Non-Newtonian Fluid. Mech., 78, pp. 227-241 ISSN: 0377-0257

Minale M. (2010) Models for the deformation of a single ellipsoidal drop: a review, Rheol. Acta, 49(8), pp. 789-806, ISSN: 0035-4511

Nam JG, Hyun K, Ahn KH, Lee SJ (2008) Prediction of normal stresses under large amplitude oscillatory shear flow. J Non-Newtonian Fluid Mech 150:1-10 ISSN: 03770257

Neidhöfer T., Siuola S., Hadjichristidis N., Wilhelm M. (2004) Distinguish Linear from Star Branched Polystyrene Solutions with Fourier Transform Rheology, Macromol. Rapid Comm., 25, pp. 1921-1926 ISSN: 1022-1336

Palierne J.F. (1990) Linear rheology of viscoelastic emulsions with interfacial tension, Rheol. Acta 29 pp. 204-214 ISSN: 0035-4511

Rallison J.M. (1984) The Deformation of Small Viscous Drops and Bubbles in Shear Flows, Ann. Rev. Fluid Mech. 16, pp. 45-66 ISSN: 0066-4189

Ramirez RW (1985) The FFT Fundamentals and Concepts. Prentice-Hall, Englewood Cliffs, NJ, ISBN: 978-0133143867

Schlatter G., Fleury G., Muller R. (2005) Fourier Transform Rheology of Branched Polyethylene: Experiments and Models for Assessing the Macromolecular Architecture, Macromolecules, 38, pp. 6492-6503 ISSN: 0024-9297

Sigillo I.; Di Santo L.; Guido S.; Grizzuti N. (1997) Comparative Measurements of Interfacial Tension in a Model Polymer Blend, Polymer Eng. and Sci. 37(9),pp. 1540-1549 ISSN: 0032-3888

H.A. Stone, Dynamics of Drop Deformation and Breakup in Viscous Fluids (1994) Ann. Rev. Fluid Mech. 26, pp. 65-102 ISSN: 0066-4189

Tucker C.L. and Moldenaers P. (2002) Microstructural Evolution in Polymer Blends, Ann. Rev. Fluid. Mech. 34, pp. 177-210 ISSN: 0066-4189

Utracki L.A. (2003) Polymer Blends Handbook, Kluwer Academic Publishers, DordrechtBoston-London ISBN: 978-1402011146

Vincze-Minya K.A., Schausberger A. (2007) Characterization of Phase Morphology of Polymer Melts(PP/PE Blends) Via Rheology, Journal of Applied Polymer Science, 105, pp. 2294-2298, ISSN: 0021-8995 
Wannaborworn S; Mackley M.R; Renardy Y. (2002) Experimental observation and matching numerical simulation for the deformation and breakup of immiscible drops in oscillatory shear. Journal of Rheology 46(5), pp. 1279-1293 ISSN: 0148-6055

Wilhelm M, Maring D, Spiess HW (1998) Fourier-transform Rheology, Rheol. Acta, 37, pp. 399-405 ISSN: 0035-4511

Wilhelm M., Reinheimer P., Ortseifer M (1999) High sensitivity Fourier Transform Rheology. Rheol. Acta 38 pp. 349-356, ISSN: 0035-4511

$\mathrm{Yu}$ W, Wang P, Zhou C (2008) General stress decomposition in nonlinear oscillatory shear flow. Journal of Rheology 53(1):215-238 ISSN: 0148-6055 


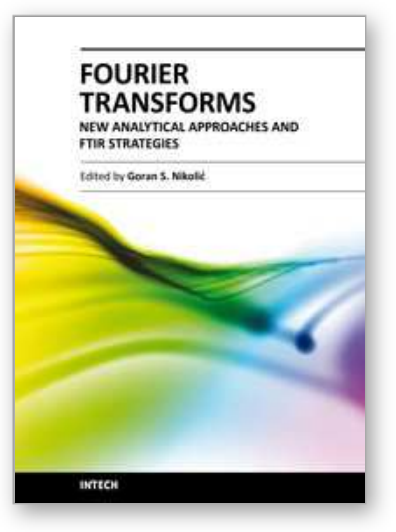

\author{
Fourier Transforms - New Analytical Approaches and FTIR \\ Strategies \\ Edited by Prof. Goran Nikolic
}

ISBN 978-953-307-232-6

Hard cover, 520 pages

Publisher InTech

Published online 01, April, 2011

Published in print edition April, 2011

New analytical strategies and techniques are necessary to meet requirements of modern technologies and new materials. In this sense, this book provides a thorough review of current analytical approaches, industrial practices, and strategies in Fourier transform application.

\title{
How to reference
}

In order to correctly reference this scholarly work, feel free to copy and paste the following:

Massimiliano Grosso and Pier Luca Maffettone (2011). Fourier Transform Rheology: A New Tool to Characterize Material Properties, Fourier Transforms - New Analytical Approaches and FTIR Strategies, Prof. Goran Nikolic (Ed.), ISBN: 978-953-307-232-6, InTech, Available from:

http://www.intechopen.com/books/fourier-transforms-new-analytical-approaches-and-ftir-strategies/fouriertransform-rheology-a-new-tool-to-characterize-material-properties

\section{INTECH}

open science | open minds

\author{
InTech Europe \\ University Campus STeP Ri \\ Slavka Krautzeka 83/A \\ 51000 Rijeka, Croatia \\ Phone: +385 (51) 770447 \\ Fax: +385 (51) 686166 \\ www.intechopen.com
}

\author{
InTech China \\ Unit 405, Office Block, Hotel Equatorial Shanghai \\ No.65, Yan An Road (West), Shanghai, 200040, China \\ 中国上海市延安西路65号上海国际贵都大饭店办公楼405单元 \\ Phone: +86-21-62489820 \\ Fax: +86-21-62489821
}


(C) 2011 The Author(s). Licensee IntechOpen. This chapter is distributed under the terms of the Creative Commons Attribution-NonCommercialShareAlike-3.0 License, which permits use, distribution and reproduction for non-commercial purposes, provided the original is properly cited and derivative works building on this content are distributed under the same license. 